\title{
Rate-compatible LDPC convolutional codes over non-gaussian noise channel
}

\author{
Qiang Liu* \\ School of Physics and Telecommunications Engineering, South China Normal University, \\ Guangzhou, Guangdong, 510006, China
}

Keywords: Non-gaussian noise, Gaussian mixture model (GMM), Ratecompatible low-density parity-check (RC-LDPC) convolutional codes.

\begin{abstract}
This paper is aimed to study the characteristics of the underwater acoustic channel with non-Gaussian noise channel. And Gaussian mixture model (GMM) is utilized to fit the background noise over the non-Gaussian noise channel. Furthermore, coding techniques which use a sequence of ratecompatible low-density parity-check (RC-LDPC) convolutional codes with separate rates are constructed based on graph extension method. The performance study of RC-LDPC convolutional codes over non-Gaussian noise channel and the additive white Gaussian noise (AWGN) channel is performed. Study implementation of simulation is that modulation with binary phase shift keying (BPSK), and iterative decoding based on pipeline log-likelihood rate belief propagation (LLRBP) algorithm. Finally, it is shown that RC-LDPC convolutional codes have good bit-rate-error (BER) performance and can effectively reduce the impact of noise.
\end{abstract}

\section{Introduction}

Non-Gaussian noise is a major impairment in many wireless communication. In signal processing, background noise is generally regarded as additive white Gaussian noise (AWGN) because of its additivity, amplitude obeying Gaussian distribution, being a kind of white noise and easy to analyze and approximate. However, the transmission of underwater acoustic signals is severely disturbed by background noise [1]. In practice, experiments conducted over the last decades show that underwater acoustic noise has the characteristics of nonGaussian [2,3]. So underwater acoustic channel is appropriate to be fitted by non-Gaussian noise channel. More non-Gaussian noise models are worth evaluating the quality of underwater acoustic communication under realistic environmental conditions. Such models are being developed $[4,5,6]$. We aim to use the Gaussian mixture model (GMM) [7] to fit the non-Gaussian noise. GMM is a linear superposition of multiple Gaussian distribution.

Channel coding plays an important part in wireless communication, which improves the reliability of signals transmitted from the source to the sink. Rate-compatible error-correcting codes are useful in various communication systems. For example, rate-compatible codes are commonly used in combination with automatic repeat requests (ARQ) to combat fading in

\footnotetext{
*Corresponding author: 2017021667@m.scnu.edu.cn
} 
wireless communications. Moreover, rate-compatible codes are a sequence of codes with different rates [8], where higher rate mother codes are embedded in the lower rate extended codes. There are two methods of constructing rate-compatible codes, puncturing $[9,10]$ and graph extension [11]. However, the punctured higher rate codes exhibit wider gaps in the capacity limit than the mother code [12]. The design of rate-compatible low-density paritycheck (RC-LDPC) block codes [13] is based on graph extension, where a high-rate base code is progressively extended to codes with lower rates. Codes produced by graph extension outperform the punctured codes over a wide range of rates.

In this paper, we use the constructed rate-compatible code family of regular low-density parity-check (LDPC) convolutional codes via graph extension method [14], which overcomes the drawbacks caused by puncturing and simplifies the optimization. LDPC convolutional codes were

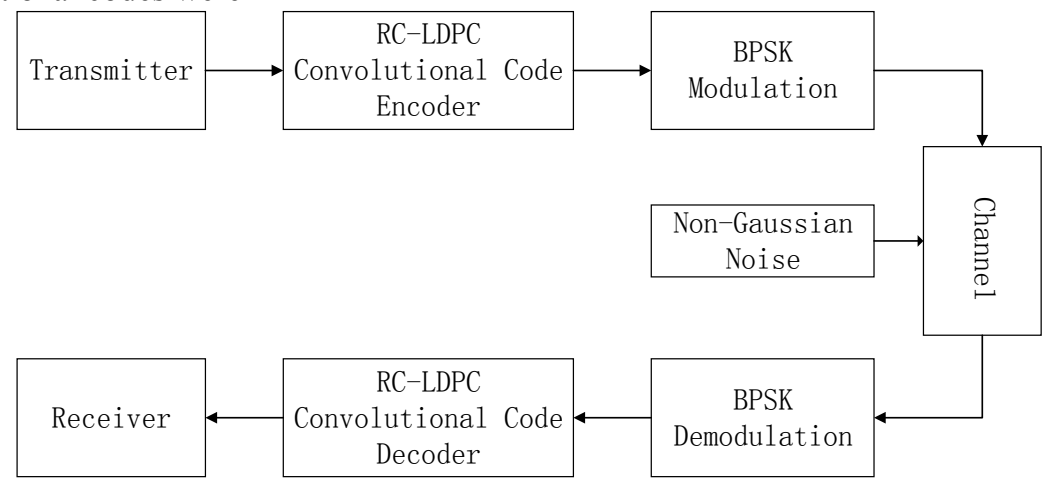

Fig. 1. Block diagram of the non-Gaussian noise channel coding system.

first proposed by A. J. Felström and K. S. Zigangirov [15] and have showed better performance than counterpart LDPC block codes [16]. Then we believe that using RC-LDPC convolutional codes constructed by graphic extension method to improve the quality of nonGaussian noise channel communication is a very effective method.

The structure of this paper is described as following. Section 2 describes non-Gaussian noise model. After that Section 3 describes communication systems and channel model. Then, Section 4 introduces the RC-LDPC convolutional codes. Section 5 continues with simulation and the characterization of RC-LDPC convolutional codes' bit-rate-error (BER) performance. And GMM is used to fit the non-Gaussian noise. The RC-LDPC convolutional code based on graph extension method is employed for non-Gaussian noise channel. Finally, the corresponding simulation results are detailed in Section 5 before offering our conclusions in Section 6.

\section{Non-gaussian noise model}

The probability model of non-Gaussian noise could be fitted by GMM. The probability density function (PDF) of GMM is the linear superposition of weight of multiple Gaussian distribution [7], which can be expressed as

$$
p(X)=\sum_{i=1}^{K} \omega_{i} g\left(X \mid \mu_{i}, \sum_{i}\right)
$$




$$
g\left(X \mid \mu_{i}, \sum_{i}\right)=\frac{1}{(2 \pi)^{\frac{N}{2}}\left|\sum_{i}\right|^{\frac{1}{2}}} \exp \left\{-\frac{1}{2}\left(X-\mu_{i}\right)^{T} \sum_{i}^{-1}\left(X-\mu_{i}\right)\right\}
$$

where $X$ represents a set of $N$-dimensional data, $K$ is the number of Gaussian distribution, $\omega_{i}$ is the mixture weight and satisfied $\sum_{i=1}^{K} \omega_{i}=1, g\left(X \mid \mu_{i}, \sum_{i}\right)$ represents the $i$-th Gaussian distribution with mean value $\mu_{i}$ and covariance matrix $\sum_{i}$.

Non-Gaussian noise is implemented by GMM through inverse function method, the noise implementation algorithm can be summarized as follows:

1. Generate two random variables $U_{1}$ and $U_{2}$. Note that $U_{1}$ and $U_{2}$ random variables are uniformly distributed from 0 to 1 .

2. Set $V_{1}=2 U_{1}-1, V_{2}=2 U_{2}-1$ and $S=V_{1}^{2}+V_{2}^{2}$. Note that $V_{1}$ and $V_{2}$ are uniformly distributed from -1 to 1 , and $S$ is uniformly distributed from 0 to 1 .

3. If $S>\omega_{i} /\left(\pi \sigma_{i}^{2}\right)$, go to step 1 , otherwise go to step 4 . Note that $\omega_{i}$ is the weight value and $\sigma_{i}^{2}$ is the variance value of the $i$-th GMM component.

4. Return the variable

$$
N_{i}=V_{1}\left(\sqrt{-\sigma_{i}^{2} \ln \left(\pi \sigma_{i}^{2} S / \omega_{i}\right)}+\mu_{i}\right) / \sqrt{S}
$$

where $\mu_{i}$ the mean value of the $i$-th GMM component.

5. Then return to the non-Gaussian noise that is superimposed by the variable $N_{i}$.

$$
N=\sum_{i=1}^{K} N_{i}
$$

where $K$ is the number of components in GMM.

\section{System and channel model}

Fig. 1 shows the non-Gaussian noise channel coding system model. In the simulation, the transmitter transmits a sequence of the information sequence, and after passing through RCLDPC convolutional code encoder with a code rate and binary phase shift keying (BPSK) modulation, it enters the non-Gaussian noise channel model for transmission. Therefore, the channel output is given by

$$
Y=X+N
$$

where $X$ is the modulated signal with BPSK, $N$ is non-Gaussian noise and is fitted by GMM.

The receiver first performs BPSK demodulation and then performs the RC-LDPC convolutional code decoding based on pipeline log-likelihood rate belief propagation (LLRBP) algorithm, and finally calculates the BER by comparing the sequence of the transmitting and the receiving 


\section{RC-LDPC convolutional codes}

The semi-infinite parity-check matrix of $\left(m_{s}, J, K\right)$ regular LDPC convolutional code with code rate $b / c$, which has $J$ ones in each column, $K$ ones in each row and $m_{s}$ is the syndrome former memory. It's known that obtaining the parity-check matrix $H_{p}$ by reorganizing the sub-matrices of the parity check matrix of the regular LDPC code [18]. Then a family of RC-LDPC convolutional codes is can be obtained from a RC-LDPC block codes family, which is constructed by using the graph extension method.

$$
H_{p}=\left[\begin{array}{cccc}
H_{0}(0) & & & \\
H_{1}(1) & H_{0}(1) & & \\
\vdots & H_{1}(2) & \ddots & \\
H_{m_{s}}(T-1) & \vdots & \ddots & H_{0}(T-1) \\
& H_{m_{s}}(0) & \ddots & H_{1}(0) \\
& & \ddots & \vdots \\
& & & H_{m_{s}}(T-2)
\end{array}\right]
$$

where matrix $H_{p}$ has $\left(2 m_{s}+1\right) \times\left(m_{s}+1\right)$ sub-matrices, each sub-matrix $H_{i}(t), i \in\left[0, m_{s}\right]$ and $\mathrm{T} \in[0$,

$$
T-1],\left(T=m_{s}+1\right) \text { is a }(c-b) \times c \text { binary matrix. }
$$

A RC-LDPC convolutional code family including $M$ member codes $C=\left\{C_{1}, \ldots, C_{k}, \ldots, C_{M}\right\}$ with different rates $R=\left\{R_{1}, \ldots, R_{k}, \ldots, R_{M}\right\}$ (in descending order) can be realized by extending the graphs. First, obtain a LDPC convolutional base code $C_{1}$ with base code rate $R_{1}=b / c$ by using the method of [18]. Let the sub-matrix of $H_{p}$ become the base sub-matrix. Second, obtain the extended LDPC convolutional code $C_{k+1}$ with code rate $R_{k+1}=b /(c+k), k \in[1, b-1]$. Each extended sub-matrix $H_{i}^{k+1}(t)$

$$
H_{i}^{k+1}(t)=\left[\begin{array}{cc}
H_{i}(t) & 0 \\
H_{E_{1, i}}^{k}(t) & H_{E_{2, i}}^{k}(t)
\end{array}\right]
$$

is obtained from basic sub-matrix $H_{i}(t)$. The extended sub-matrices $H_{E_{1, i}}^{k}(t)$ and $H_{E_{2, i}}^{k}(t)$ are obtained from choosing the corresponding elements of $H_{E_{1}}^{k}$ and $H_{E_{2}}^{k}$ in detail in [14]. Then we get each sub-matrix $H_{i}^{k+1}(t)$ of matrix $H_{p}^{k+1}$ corresponding to LDPC convolutional code $C_{k+1}$. So a member of RC-LDPC convolutional code family is obtained.

$$
H_{E_{1}}^{k}=\left[\begin{array}{cccccc}
I_{1} & I_{1} & 0 & \cdots & 0 & 0 \\
0 & 0 & I_{1} & \cdots & 0 & 0 \\
\vdots & \vdots & \vdots & \cdots & \vdots & \vdots \\
0 & 0 & 0 & \cdots & 0 & I_{1}
\end{array}\right]
$$




$$
H_{E_{2}}^{k}=\left[\begin{array}{ccccc}
H_{e x t} & 0 & \cdots & 0 & 0 \\
I_{1} & H_{e x t} & \cdots & 0 & 0 \\
\vdots & \vdots & \cdots & \vdots & \vdots \\
0 & 0 & \cdots & I_{1} & H_{e x t}
\end{array}\right]
$$

where $I_{1}$ is an $\left(m_{s}+1\right) \times\left(m_{s}+1\right)$ identity matrix, $H_{\text {ext }}$ matrix is the parity bits part of the $\left(m_{s}+1\right) \times 2\left(m_{s}+1\right)$ parity-check matrix with a code rate $R=1 / 2$ obtained according to [18]. And 0 is an $\left(m_{s}+1\right) \times\left(m_{s}+1\right)$ zero matrix.

The binary parity-check matrix of a rate $R=b /(c+k), k \in[1, b-1]$ RC-LDPC convolutional code is denoted as $H_{p}^{k}$, a binary codeword sequence $v=\left[v(0), v(1), \ldots, v(t), \ldots, v\left(2 m_{s}+1\right)\right]$ must satisfy

$$
H_{p}^{k} v^{T}=0^{T}
$$

where $v(t), t \in\left[0,2 m_{s}+1\right]$ of length $(c+k)$. In order to simplify the implementation of the encoding, the first $b$ bits of each convolutional code group are copies of the information bits, and the parity-check matrix $H_{p}^{k}$ is used to get the check bits of the corresponding information bits by Eq. 10. Then we can get the codeword $v$ after the information sequence is encoded. Because parity-check matrices of RC-LDPC convolutional codes are sparse, they can be decoded using the same pipeline LLRBP decoding algorithm [15] as that used for LDPC block codes.

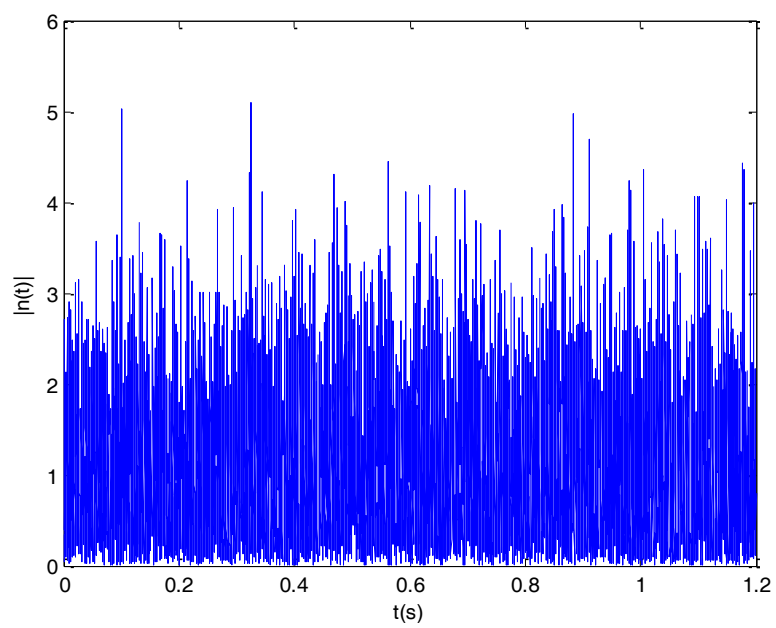

Fig. 2. The GMM noise given by $\mathrm{p}(\mathrm{X})=0.8143 \mathrm{CN}(\mathrm{X} ; 0.0047,0.655)+0.1857 \mathrm{CN}(\mathrm{X} ; 0.0328,2.5236)$.

\section{Simulations and experiments}

Study implementation of simulation over non-Gaussian noise channel and AWGN channel is performed. The PDF of the non-Gaussian noise sample which collected in the South China 
Sea is used by Eq. 11. Fig. 2 shows the non-Gaussian noise sample collected in the South China Sea is fitted using a two-component GMM. The PDF of the noise is given by [17]

$$
p(X)=0.8143 C N(X ; 0.0047,0.655)+0.1857 C N(X ; 0.0328,2.5236)
$$

where $C N(X ; 0.0047,0.655)$ is a circular Gaussian distribution with mean value 0.0047 and variance $0.655, C N(X ; 0.0328,2.5236)$ is a circular Gaussian distribution with mean value 0.0328 and variance 2.5236 , and weight values are 0.8143 and 0.1857 respectively. The PDF of $C N\left(X ; \mu, \sigma^{2}\right)$ is

$$
C N\left(X ; \mu, \sigma^{2}\right)=\frac{1}{\pi \sigma^{2}} \exp \left\{-\frac{|X-\mu|^{2}}{\sigma^{2}}\right\}
$$

The encoder is based on the regular RC-LDPC convolutional code family with code rates varied from $7 / 11$ to $7 / 17$ constructed by the graph extension method, where the syndrome former memory $m_{s}=67$ and the base code rate $R_{1}=7 / 11$. The simulation modulation mode is BPSK modulation and using a number of processors $I=50$ of the pipeline LLRBP decoding algorithm. Furthermore, under non-Gaussian noise and AWGN, the BER performance curves of RC-LDPC convolutional codes with different code rates are shown in Fig. 3 and Fig. 4. It has been showed that as the value of $k$ increases, the performance of the corresponding RC-LDPC convolutional code becomes more excellent under nonGaussian noise and AWGN. The performance of rate $R=7 / 14, m_{s}=67$ LDPC convolutional codes are much better than the concatenated code, which is a combination of inner code Hadamard code H(20,5) and outer code 32-ary $(620,310)$ LDPC code of [17]. And the RC-LDPC convolutional code family has better performance than the constructed LDPC convolutional base code with a code rate $R=7 / 11$.

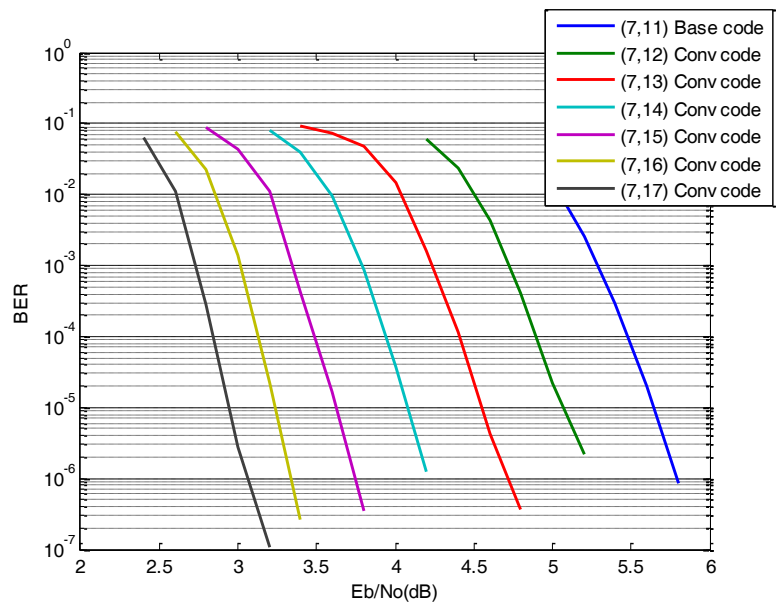

Fig. 3. BER performance of RC-LDPC convolutional code family over the non-Gaussian noise channel. 


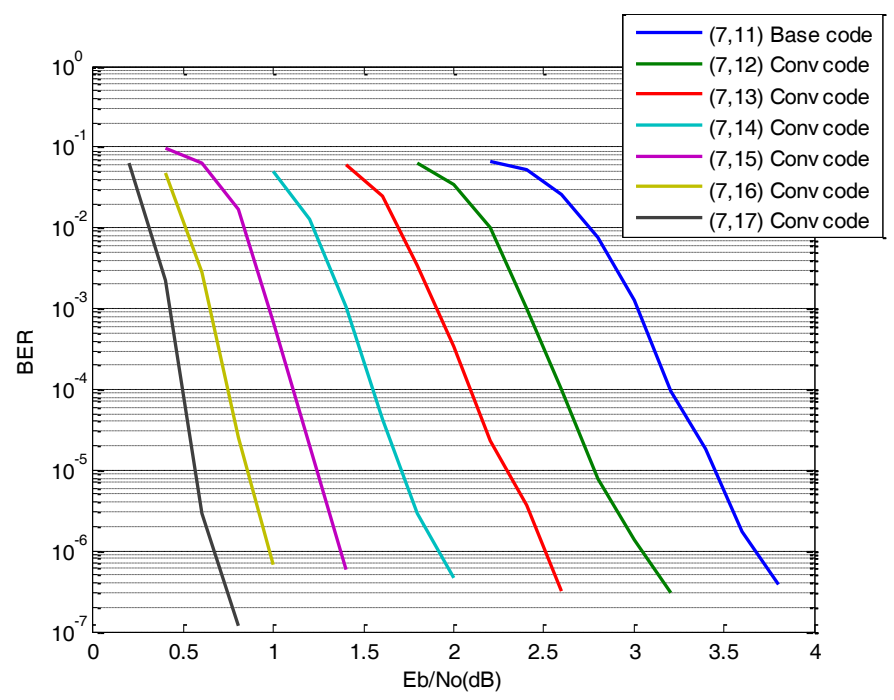

Fig. 4. BER performance of RC-LDPC convolutional code family over the AWGN channel.

\section{Conclusion}

In this paper, underwater acoustic channel is characterized as the non-Gaussian noise channel. And the presence of non-Gaussian noise is modeled as GMM. The RC-LDPC convolutional code family with the syndrome former memory $m_{s}=67$ are constructed based on the graph extension method. Study implementation of simulation is acquired by RC-LDPC convolutional code family over communication system with BPSK and iterative decoding by LLRBP algorithm based on the Tanner graph. And the simulation results show that the error correcting performance of RC-LDPC convolutional codes with AWGN has better improvements compared to the concatenated code. In addition, the simulation results also show that RC-LDPC convolutional code family is constructed based on graph extension, can achieve reliable transmission with a BER less than $10^{-5}$ under non-Gaussian noise channel and AWGN channel. Finally, we conclude that the RC-LDPC convolutional code family based on GMM can effectively reduce the influence of the non-Gaussian noise in the communication system.

\section{References}

1. G.M. Wenz, Acoustic ambient noise in the ocean: Spectra and sources, The Journal of the Acoustical Society of America. 34(1962) 1936-1956.

2. P.L Brockett, M. Hinich, G.R Wilson, Nonlinear and non-Gaussian ocean noise, The Journal of the Acoustical Society of America. 82(1987) 1386-1394.

3. R.J Webster, Ambient noise statistics, IEEE Transactions on Signal Processing. 41(1993) 2249-2253.

4. J. Aparicio, A. Jiménez, J. Ureña and F.J Álvarez, Realistic modeling of underwater ambient noise and its influence on spread-spectrum signals, OCEANS 2015-Genova. (2015) 1-6. 
5. C.Y. Nguyen, H.V. Do, V.D. Nguyen and H.A. Muzamane, Underwater ambient noise model and verification in the underwater OFDM system, 2017 International Conference on Information and Communications (ICIC). (2017) 233-239.

6. M. Rahmati, D. Pompili, UNISeC: Inspection, Separation, and Classification of Underwater Acoustic Noise Point Sources, IEEE Journal of Oceanic Engineering. 43(2018) 777-791.

7. J.G. Liu, Xianbin Wang, J. Nadeau and Hai Lin, Modulation classification based on Gaussian mixture models under multipath fading channel, 2012 IEEE Global Communications Conference (GLOBECOM). (2012) 3970-3974.

8. Z. Si, R. Thobaben, M. Skoglund, Rate-Compatible LDPC Convolutional Codes Achieving the Capacity of the BEC, IEEE Transactions on Information Theory. 58(2012) 4021-4029.

9. C. Hsu, A. Anastasopoulos, Capacity Achieving LDPC Codes Through Puncturing, IEEE Transactions on Information Theory. 54(2008) 4698-4706.

10. M. El-Khamy, J. Hou, N. Bhushan, Design of rate-compatible structured LDPC codes for hybrid ARQ applications, IEEE Journal on Selected Areas in Communications. 27(2009) 965-973.

11. M.R. Yazdani, A.H. Banihashemi, On construction of rate-compatible low-density Parity-check codes, IEEE Communications Letters. 8(2004) 159-161.

12. R. Asvadi, A. H. Banihashemi, A Rate-Compatible Puncturing Scheme for Finite-Length LDPC Codes, IEEE Communications Letters. 17(2013) 147-150.

13. N. Jacobsen, R. Soni, Design of Rate-Compatible Irregular LDPC Codes Based on Edge Growth and Parity Splitting, 2007 IEEE 66th Vehicular Technology Conference. (2007) 1052-1056.

14. L. Mu, C. Liang, Z. Liu and D. Pan, Construction of regular rate-compatible LDPC convolutional codes, China Communications. 13(2016) 97-102.

15. A. Jimenez Felstrom, K. S. Zigangirov, Time-varying periodic convolutional codes with low-density parity-check matrix, IEEE Transactions on Information Theory. 45(1999) 2181-2191.

16. R. Gallager, Low-density parity-check codes, IRE Transactions on Information Theory. 8(1962) 21-28.

17. D. Li, Y. Wu, M. Zhu, Nonbinary LDPC code for noncoherent underwater acoustic communication under non-Gaussian noise, 2017 IEEE International Conference on Signal Processing, Communications and Computing (ICSPCC). (2017) 1-6.

18. L. Mu, X. Liu, C. Liang, Improved construction of LDPC convolutional codes with semirandom parity-check matrices, Science China(Information Sciences). 02(2014) 37-46. 